\title{
Fetal ovarian cyst: Prenatal diagnosis and successful postnatal surgical management
}

\author{
Georgina Álvarez Medina ${ }^{1 *}$ and Laura Tabares Saéz ${ }^{2}$ \\ ${ }^{1}$ Master en Atención Integral a la Mujer, Especialista de Ginecobstetricia grado, Cuba \\ ${ }^{2}$ Residente de 3er año de Ginecobstetricia, Cuba
}

Submission: November 04, 2019; Published: November 07, 2019

*Corresponding author: Georgina Álvarez Medina, Master en Atención Integral a la Mujer, Especialista de Ginecobstetricia grado, Cuba

\begin{abstract}
Objective: Demostrar que el diagnóstico temprano de los tumores fetales interviene en el resultado exitoso

Introducción: Los quistes ováricos fetales son el tumor abdominal más frecuente en las recién nacidas siendo la tercera causa de quistes intrabdominales, después de los de sistemas renal y gastrointestinal. Se puede sospechar su presencia en la ecografía cuando se observa una tumoración de aspecto quístico de estructura regular localizado en la parte inferior y lateral del abdomen, del tracto intestinal o urinario en un feto femenino sin anomalías. Presentan complicaciones agudas y a largo plazo. La más frecuente es la torsión anexial que presenta dificultades diagnósticas en la etapa neonatal. El diagnóstico prenatal es fundamental para el manejo oportuno de las pacientes. Existen diferentes alternativas terapéuticas donde la cirugía mínimamente invasiva y conservadora juega un importante rol. Presentación del caso: Se presenta el caso de una paciente de 37 años primigesta, a la que durante la evaluación ecográfica se le observó la presencia de una tumoración de apariencia blanda en la parte inferior derecha del abdomen correspondiente a un quiste ovárico fetal claramente identificado y speared de la vejiga fetal.
\end{abstract}

Conclusiones: La paciente fue sometida a cesárea obteniéndose una recién nacida femenina a la cual a los dos días de nacida se intervino quirúrgicamente por mínimo acceso y se resolvió exitosamente gracias a un manejo multidisciplinario.

Keywords: Quiste ovárico fetal; Diagnóstico prenatal; Torsión ovárica neonatal, Fetal ovary cystic, Prenatal diagnosis, Adnexal twisted

\section{Fetal Ovarian Cyst}

Prenatal diagnosis and successful postnatal surgical management.

\section{Objective}

To demonstrate the early diagnosis abdominal fetal tumor, successfully intervention result. Ovarian cyst the most frequent abdominal tumor among female newborns and the third cause of intrabdominal cyst, right after those of the renal system and gastrointestinal. Whenever a tumor of cyst aspect of regular structure located in the lower part of the body and the side part of the abdomen, of the intestinal tract or urinary of the female fetus is viewed on the ultrasound scan, its presence can be suspected. Acute and long term complications are present. The most frequent one is adnexal twisted which are very difficult for diagnosis in the fetal period. Prenatal diagnosis is fundamental for opportune management of patients. There are different therapeutic alternatives, and the Minimally Invasive Surgery and conservative treatment have an important role. A case of a 37-year-old patient of first gestation is presented, who during the ultrasound scan evaluation was found a tumor in the right inferior part of the fetus' abdomen, with a soft aspect corresponding of a fetal ovary tumor separated of fetal urinary bladder. The female baby was born by cesarean section, and when she was two days old, she had a surgical Minimally Invasive Surgery intervention, and the problem was solved by multidisciplinary teams participating.

\section{Introduction}

La presencia de quistes ováricos (Q0) en fetos y recién nacidos es muy frecuente. El 34\% de los neonatos femeninos presentan quistes ováricos de al menos $10 \mathrm{~mm}$ según el resultado de autopsias; se estima ecográficamente una incidencia de uno cada 2625 recién nacidos femeninos [1]. El diagnóstico de ellos se ha incrementado gracias al uso rutinario de ultrasonografía en el control prenatal. Esta enfermedad era prácticamente desconocida antes de 1975 en que Valenti 1 describió por primera vez su diagnóstico por ultrasonido. El uso rutinario de la ecografía durante la gestación ha incrementado la detección prenatal de quistes ováricos. 1 Su etiología no está aún definida, pero se consideran resultado de la estimulación folicular ovárica por los estrógenos maternos, gonadotropina coriónica placentation y gonadotropina fetal. [2,3]. 
En el último trimestre del embarazo el feto está bajo la influencia de altas tasas de esteroides placentarios. Al nacer, estos disminuyen bruscamente produciéndose liberación de GnRh, con aumento secundario de las hormonas folículo estimulante y luteinizante (FSH y LH) en el feto femenino, principalmente la FSH que se mantiene en niveles detectables hasta los dos a tres años de vida. De esta manera el ovario fetal presenta crecimiento folicular intra útero, folículos primarios desde las 20 semanas de gestación y folículos de Graaf desde las 32 semanas, presentes en el $40-60 \%$ de los ovarios de las recién nacidas. A veces estos folículos crecen y forman quistes ováricos prenatalmente, procesos autolimitados al desaparecer la acción esteroidea materna. En esta etapa de la vida los quistes ováricos tienen una frecuencia de 30-70\% según la edad gestacional. Frecuentemente son unilaterales. Solo $20 \%$ de estos son mayores de 9 milímetros, y se consideran patológicos si son mayores de 2 centímetros [3]. También se pueden presentarse en algunas complicaciones maternas tales como: preeclampsia, diabetes mellitus e isoinmunización Rh, debido al incremento de la gonadotropina placentaria como: hipotiroidismo o hiperplasia adrenal congénita por déficit de la 21 hidroxilasa o de la 11- $\beta$ hidroxilasa.

Todas estas teorías se relacionan con los cambios hormonales que, típicamente, se producen durante el embarazo. Postnatalmente, se manifiestan por una masa abdominal palpable que puede ser asintomática o presentar sintomatología relacionada a las complicaciones del quiste [2]. Dichas complicaciones ocurren en el 30 a $40 \%$ de casos, siendo más frecuente la torsión ovárica que puede provocar la pérdida del ovario 4,5 La ecografía permite diferenciar los quistes ováricos como simples y complejos.

La conducta a seguir depende del momento que se hace el diagnóstico, las características del quiste y la presencia de complicaciones. En el neonato, la sintomatología es de aparición tardía e inespecífica lo que determina que el diagnóstico precoz sea un desafío. Objetivo: Presentar el caso poco frecuente, de una recién nacida con diagnóstico prenatal de un quiste de ovario complicado por su torsión después de su nacimiento.

\section{Clinical Case Report}

Se trata de paciente femenina de 37 años de edad, raza blanca, con antecedente de seguimiento por consulta de infertilidad por presentar diagnóstico de climaterio precoz en la cual se aplicó una de las técnicas de reproducción asistida de alta complejidad Fertilización in vitro/ Ovodonación (FIV/OD). Se prescribió terapia sustitutiva por fallo ovárico con valerianato de estradiol hasta las 12,5 semanas de gestación. Gestante con grupo sanguíneo A+, con antecedente de conización por presentar NIC II y antecedentes familiares de ambos padres con hipertensión arterial.

\section{Obstetric History}

G1 P0 A0, Fecha de la última menstruación 14-2-2008. Durante el seguimiento prenatal se observa como datos positivos recogidos en la historia clínica un índice de masa corporal IMC a la captación catalogándose como sobrepeso, con una curva de tensión arterial sin descenso fisiológico del segundo trimestre, con cifras sistólicas de $130 \mathrm{mmHg}$ y diastólicas de $80 \mathrm{mmHg}$ a la captación.

A las 33.5 semanas de edad gestacional se constatan en consulta externa de FIV cifras elevadas de tensión arterial $(140 / 100 \mathrm{mmHg})$ de manera que se decide ingreso hospitalario. Durante su estadía se mantuvo asintomática, con exámenes complementarios de laboratorio con valores normales. (Ácido úrico ,creatinina, coagulograma completo, transaminasa glutámico pirúvica, transaminasa glutámico oxalacetica) Se le realiza fondo de ojo que informa vasos con ligera tortuosidad vascular y papila normal, lo cual sugiere posible hipertensión arterial crónica para lo cual nunca llevó tratamiento antihipertensivo.

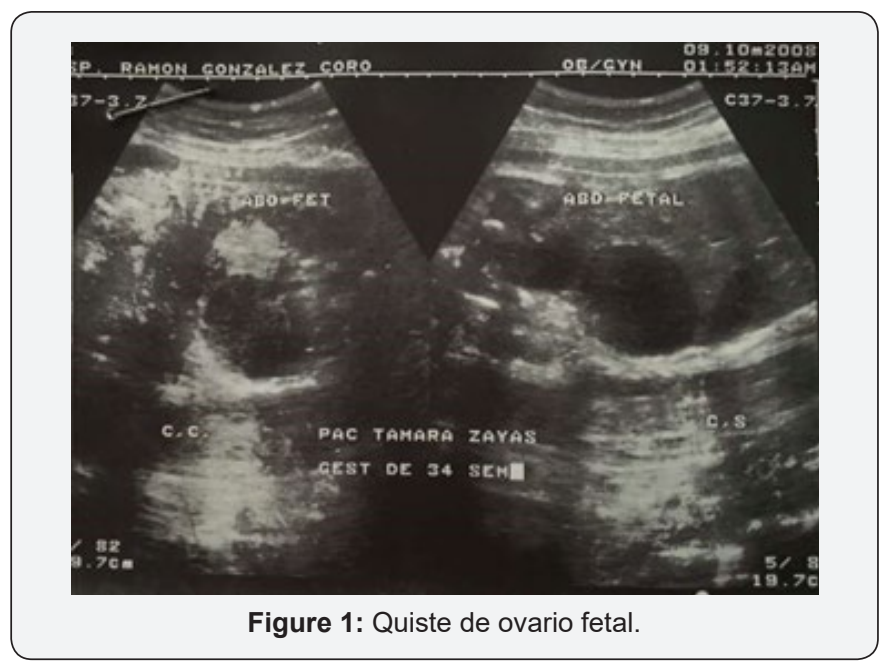

A las 34 semanas de edad gestacional se le realiza ultrasonido que informa imagen de más menos $5 \mathrm{~cm}$ de aspecto quístico en abdomen fetal (Figure 1-3). Se decide repetir en consulta de Genética (a las 34,4 semanas) informando imagen quística de $48 \times 58 \mathrm{~mm}$ de paredes lisas y contenido liquido en ovario izquierdo, se sugiere reevaluar a las 35 semanas de gestación, se realiza e informa tumoración quística por encima de la vejiga de 56 x $45 \mathrm{~mm}$ sin elementos en su interior, no existe compromiso de asas intestinales,

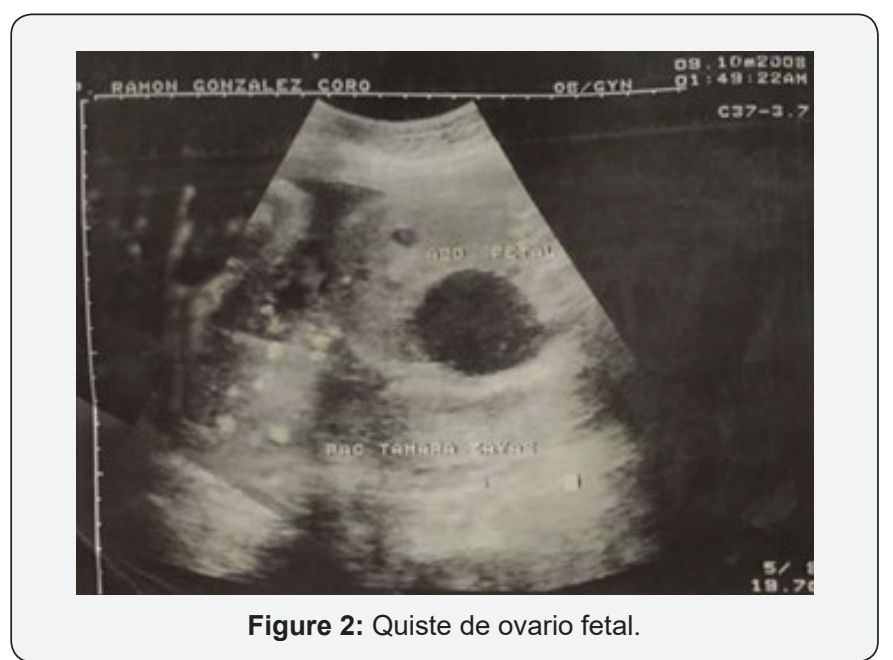




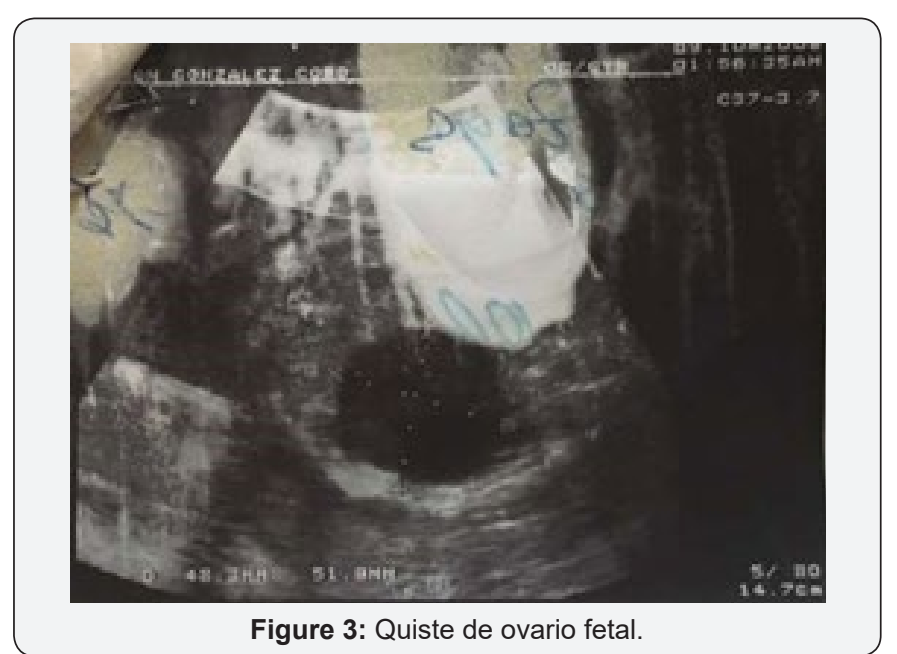

Impresión diagnóstica: ID: Quiste de ovario versus quiste del mesenterio fetal. Durante todo el seguimiento de la gestante, las pruebas de bienestar fetal realizadas se encontraron dentro de parámetros normales. (cardiotocografia simple, ultrasonido, perfil biofísico fetal y conteo de movimiento fetales) El feto tuvo un crecimiento acorde a su edad gestacional el cual se mantuvo siempre por encima del 50 percentil.

Se decide ante esta paciente tratada por fertilización invitro con edad gestacional de 39,4 semanas en la que se había realizado el diagnóstico prenatal de quiste de ovario fetal, realizar cesárea primitiva, la cual se efectúa sin complicación. Cesárea (18/11/2008) Recién nacido femenino, 3160 gramos de peso, Apgar 9/9, $50 \mathrm{~cm}$ de talla, $34 \mathrm{~cm}$ de circunferencia cefálica, $32 \mathrm{~cm}$ de circunferencia torácica, $29 \mathrm{~cm}$ de circunferencia abdominal. Al examen físico del abdomen se constata blando, con tumoración palpable en flanco y fosa iliaca izquierda de más menos cuatro centímetros, el cual se comprueba mediante ultrasonido abdominal fetal que informa imagen ovárica redondeada de 58x43x$47 \mathrm{~mm}$ con elementos celulares en su interior.

Por tal motivo se decide su remisión al Hospital Pediátrico Ángel Arturo Aballí en La Habana, donde se le realiza a los dos días de nacida intervención quirúrgica por mínimo acceso confirmando durante el acto quirúrgico la presencia de quiste de ovario izquierdo de más menos $5 \mathrm{~cm}$ al cual se le realiza la enucleación además de trompa izquierda torcida la cual es amputada y un útero aumentado de tamaño con relación a su edad. La cirugía termina sin complicaciones y la recién nacida presentó una adecuada recuperación, siendo egresada a los tres días del postoperatorio.

\section{Discussión}

Los Q0 representan la masa abdominal quística más común en fetos y neonatos femeninos [3]. Desde el primer reporte en 1975 [1], el diagnóstico prenatal se ha incrementado gracias a la incorporación del examen ecográfico de rutina por parte de especialistas capacitados y al avance tecnológico que permite obtener imágenes de mejor resolución. Su incidencia se estima en uno cada 2625 recién nacidos femeninos. 4 El diagnóstico diferencial de una masa abdominal quística en un feto o neonato del sexo femenino incluye: anomalías gastrointestinales (quistes de duplicación intestinal, quistes mesentéricos), anomalías del tracto genitourinario (hidro-metro-colpos, quistes renales, del uréter, entre otras), linfagioma, meningocele anterior, entre otros, [1,5] siendo el diagnóstico diferencial más difícil en casos de duplicación intestinal.

Como en este caso que se presenta, la mayoría de los QO se diagnostican en fetos femeninos en embarazos normales son simples, unilaterales y se detectan durante el tercer trimestre de la gestación [6,7]. La mayor parte de ellos son funcionales, es decir, se forman a partir del tejido ovárico fetal en respuesta al propio estimulo hipofisario de la FSH, estrógenos maternos y gonadotrofina coriónica placentaria (HCG) [2,8]. Sin embargo, en raras ocasiones puede corresponder a teratomas maduros e inmaduros [8]. Se puede asociar a otras afecciones fetales como hipotiroidismo o maternas que aumentan la secreción de HCG. La estimulación ovárica en mujeres infértiles como posible causa de los quistes ováricos en la recién nacida sólo ha sido referida por Van der Zee. 19 Sólo los QO mayores a $2 \mathrm{~cm}$ deben considerarse patológicos [7-9].

El pronóstico de los QO es variable. Pueden presentar torsión o alguna complicación secundaria a una hemorragia intra quística o a efecto de masa produciendo compresión. La incidencia de la torsión del quiste puede ser tan elevada que alcance 50-78\%, con $40 \%$ de ocurrencia en la etapa prenatal $[7,8]$.

Los quistes de gran tamaño pueden provocar en la vida fetal polihidramnios (10-20\%), hipoplasia pulmonar (ambas asociaciones pueden ser explicadas por la presencia de grandes quistes que disminuyen el espacio intraabdominal fetal, produciendo obstrucción intestinal y compresión del diafragma), ascitis, peritonitis por rotura y distocia por aumento del diámetro abdominal $[10,11]$. La mayoría de los QO simples desaparecen espontáneamente al disminuir las hormonas de origen materno-placentario y la actividad del eje hipotálamohipofisario-gonadal fetal [2]. La torsión del pedículo ovárico no solo puede provocar la pérdida de este, también puede poner en riesgo la vida de la paciente. Si existe la sospecha de torsión, se debe considerar la interrupción electiva del embarazo cerca de su término o cuando se compruebe la madurez pulmonar fetal para aumentar las posibilidades de preservar la función ovárica en fetos con quistes ováricos bilaterales.

La presencia de un quiste ovárico fetal en la etapa prenatal no constituye siempre una indicación para realizar una cesárea. Dentro de las complicaciones, la presencia de extenso tejido necrótico genera una reacción inflamatoria con desarrollo de adherencias intestinales que pueden provocar obstrucción intestinal/urinaria secundaria, perforación intestinal y peritonitis $[12,13]$. La rotura puede provocar hemorragia severa con shock hipovolémico. Sólo se ha reportado un caso de muerte en la literature [14]. 
El diagnóstico del quiste ovárico fetal es principalmente ecográfico y se basa en la presencia de cuatro criterios: [10] sexo femenino, estructura quística de contorno regular fuera de la línea media, tracto urinario y gastrointestinal de apariencia normal. La ecografía también nos permite establecer si el QO está complicado. Los no complicados son de pared fina y de contenido anecogénico, los complicados por torsión o hemorragia intraquística se presentan con contenido hiperecogénico o con tabiques o pared fina hiperecogénica o con nivel líquido y detritos en su interior [10]. Este último es el signo más característico de torsión, tal como se presentó en la paciente que se presenta. Existen diferentes alternativas de tratamiento que van desde una conducta quirúrgica radical a una expectante, pasando por punción percutánea pre y postnatal. En las últimas décadas se ha reunido experiencia en las diferentes terapias por lo que el manejo es aún motivo de discusión.

En general se puede afirmar que el manejo postnatal de los QO depende de la existencia de complicaciones y de su tamaño. Existe consenso en adoptar una conducta expectante con control seriado ecográfico si el QO es simple y mide menos de $5 \mathrm{~cm}$, ya que la gran mayoría desaparece de forma espontánea $(50 \%$ al mes de vida, $75 \%$ a los 2 meses y $90 \%$ a los 3 meses) [8]. Prácticamente no existe riesgo de malignidad; sin olvidar que se han reportado casos de torsión en quistes de dos centímetros $[7,8]$.

Los QO simples mayores a cinco centímetros pueden ser evacuados de su contenido por punción percutánea para acelerar su resolución y disminuir el riesgo de torsión $[15,16]$. Los quistes ováricos fetales y maternos pueden complicarse con torsión, hemorragia intraquística, ruptura con hemorragia intrabdominal y causar distocia del trabajo de parto $[2,4]$. Los quistes de ovario fetales, de gran tamaño pueden ocasionar distrés respiratorio, hipoplasia pulmonar, polihidramnios, perforación intestinal y fallo hepático. 8 Mientras algunos autores afirman que el riesgo de torsión se relaciona con el tamaño del quiste, otros consideran que se relaciona con la longitud del pedículo. 5.9 Esta complicación puede producirse en el feto femenino o después de su Nacimiento [4].

En el recién nacido es difícil establecer clínicamente el diagnóstico precoz, ya que su sintomatología es tardía e inespecífica. La torsión neonatal de un quiste ovárico cursa con síntomas inespecíficos como: llanto e irritabilidad, pobre lactancia, vómitos, febrícula y distensión abdominal [5]. Otro signo que puede aparecer es la taquicardia fetal, probablemente causada por irritación peritoneal. El diagnóstico prenatal de ellos permite un control ecográfico seriado postparto para detectar a tiempo la ocurrencia de esta complicación. Si en el período neonatal los quistes presentan síntomas y/o características ecográficas de torsión, no hay duda que se debe realizar cirugía para preservar la gónada y evitar mayores complicaciones.

Existe controversia respecto al manejo de quistes complejos; algunos recomiendan tratamiento conservador con seguimiento clínico y ecográfico en casos seleccionados: quistes de origen ovárico, niveles de alfafetoproteína y gonadotropina coriónica humana subunidad $\beta$ normales y ausencia de sintomatología. $[2,15,16]$. Otros autores recomiendan tratamiento quirúrgico para disminuir el riesgo de torsión y otras complicaciones, y así asegurar una mayor preservación de tejido ovárico [1,14].

En el tratamiento de los quistes ováricos, se recomienda la preservación quirúrgica de la mayor cantidad de tejido gonadal posible para asegurar el desarrollo puberal y conservación de la fertilidad $[3,14]$. Esta debe ser lo más conservadora posible, independiente de la apariencia macroscópica, ya que esta no refleja el grado de necrosis del ovario. Esta afirmación se basa en el hallazgo de folículos en estudios histológicos de piezas quirúrgicas demostrando que la ooforectomía en estos casos es excesiva. Por otra parte no existen reportes de complicaciones tromboembólicas posteriores a la cirugía conservadora. La liberación de adherencias, revertir la torsión, vaciamiento y el desteche de su cápsula serían suficientes para asegurar su solución [12,17].

La vía laparoscópica ofrece ventajas sobre la técnica abierta ya que permite confirmar el diagnóstico en caso de dudas, existe menos dolor postoperatorio, la reincorporación más rápida a la alimentación y la incidencia más baja de adherencias postoperatorias. Este último punto es especialmente importante en la preservación de la fertilidad en una paciente que potencialmente ha perdido una gónada [16].

En relación a los Q0 complejos asintomáticos existen diferentes opiniones. Galinier y cols [18], con la mayor serie retrospectiva publicada, señalan que la cirugía neonatal inmediata solo se justifica si han treasured menos de 1-2 semanas desde el momento del diagnóstico de torsión, pasado este plazo la posibilidad de encontrar tejido viable es prácticamente cero y los riesgos anestésicos quirúrgicos muy altos. Bagolan y cols., 12 con la mayor serie prospectiva publicada, plantean que todos los QO fetales complicados que persisten al momento del nacimiento debieran resolverse quirúrgicamente por el desarrollo de adherencias y sus consecuencias.

Se recomienda seguimiento ecográfico a todas las pacientes evolucionadas de forma conservadora para evidenciar la resolución y la visualización de ambos ovarios. La experiencia acumulada indica que la torsión de un quiste durante la vida intrauterina es muy frecuente y que la conducta expectante puede llevar a la pérdida del ovario asociada con el desarrollo de otras complicaciones. Al considerar estos hechos, diferentes autores se motivaron hace poco más de una década a realizar punciones evacuadoras "in útero". Actualmente, basados en estudios prospectivos y retrospectivos, $[6,12,18]$ se señala que el vaciamiento prenatal de QO mediante punción guiada por ultrasonido disminuye en forma significativa el riesgo de torsión, la necesidad de cirugía neonatal y contribuye a establecer el diagnóstico definitivo a través de la medición de estradiol de la muestra. Es considerada una alternativa válida, efectiva y sin complicaciones en centros que cuentan con experiencia en cirugía fetal. 
Resulta interesante compartir y presentar este caso por su asociación con el tratamiento hormonal sustitutivo que recibió la paciente para lograr el producto de la concepción. Además permite comprender la importancia del diagnóstico y manejo oportuno de los QO fetales, confirmando la necesidad del trabajo coordinado entre diferentes especialidades, en beneficio de nuestras pacientes.

\section{Conclusión}

Mediante un adecuado control prenatal con ecografía de alta resolución se logró establecer el diagnóstico prenatal precoz de un quiste de ovario complicado por su torsión en una recién nacida de dos días de vida que fue intervenida por cirugía conservadora de mínimo acceso según las recomendaciones establecidas en la literatura, antes que el daño fuera irreversible y la situación clínica empeorara. Se logró una rápida recuperación y un mínimo impacto sobre el pronóstico reproductivo de la paciente.

\section{References}

1. Valenti C, Kassner EG, Yermakov V, Cromb A (2012) Antenatal diagnosis of a fetal ovarian cyst. Am J Obstet Gynecol 5: 216-219.

2. Grumbach MM, Kaplan SL (2013) Fetal pituitary hormones and the maturation of central nervous system regulation of anterior pituitary function. In: Gluck L (Ed), Modern Perinatal Medicine, Year Book, Chicago, pp. 247-256.

3. Armas A, Taboada P, Pradillos J (2010) Tratamiento quirúrgico de los quistes ováricos perinatales. Cir Pediatr 23: 225-228.

4. Templeman C (2004) In Training Ovarian Cysts. J Pediatr Adolesc Gynecol 17: 297-298.

5. Brandt M, Helmrath M (2005) Ovarian cysts in infants and children. Semin Pediatr Surg 14(2): 78-85.

6. Akin M, Akin L, Özbek S, Tireli G, Kavuncuoğlu S, et al. (2010) Fetal-Neonatal ovarian cysts. Their monitoring and management: retrospective evaluation of 20 cases and review of the literature. J Clin Res Ped Endo 2(1): 28-33.
7. Noia G, Riccardi M, Visconti D, Pellegrino M, Quattrocchi T, et al. (2012) Invasive fetal therapies: Approach and results in treating fetal ovarian cysts. J Matern Fetal Neonatal Med 25(3): 299-303.

8. Bryant AE, Laufer MR (2004) Fetal ovarian cysts: incidence, diagnosis and management. J Reprod Med 49(5): 329-337.

9. Jafri S, Bree R, Silver T, Ouimette M (2014) Fetal ovarian cysts: sonographic detection and association with hypothyroidism. Radiology $150(3): 809-812$

10. Nussbaum A, Sanders R, Hartman D (2013) Neonatal ovarian cyst: Sonographic pathologic correlation. Radiology 168(3): 817-821.

11. Hasiakos D, Papakonstantinou K, Bacanu A (2008) Clinical experience of five fetal ovarian cysts: diagnosis and follow-up. Arch Gynecol Obstet 277(6): 575-578.

12. Bagolan P, Giorlandino C, Nahom A (2012) The management of fetal ovarian cysts. J Pediatr Surg 37(1): 25-30.

13. Koç E, Türkyilmaz C, Atalay Y, Baskar C, Bideci A, et al. (2012) Neonatal ovarian cyst associated with intestinal obstruction. Indian J Pediatr 64(4): 555-557.

14. Kasian GF, Taylor BW, Sugarman RG, Nyssen JN (2013) Ovarian torsion related to sudden infant death. CMAJ 135(12): 1373-1375.

15. Widdowson DJ, Cook C (2013) Neonatal ovarian cysts: therapeutic dilemma. Arch Dis Child 63(7 Spec No): 737-742.

16. Bailez M (2009) Masas anexiales en el recién nacido, periodo peripuberal y teratomas maduros del ovario. Enfoque clínico actual, roly alcances del tratamiento por videolaparoscopia. Rev Med Clin Condes 20(6): 835-839.

17. Bayer AI, Wiskind AK (1994) Adnexal torsion: can the adnexa be saved? Am J Obstet Gynecol 171(6): 1506-1510.

18. Galinier P, Carfagna L, Juricic M, Lemasson F, Moscovici J, et al. (2008) Fetal ovarian cysts management and prognosis: a report of 82 cases. J Pediat Surg 43(11): 2004-2009.

19. van der Zee DC, van Seumeren IG, Bax KM, Rövekamp MH, ter Gunne A] et al. (2014) Laparoscopic approach to surgical management of ovarian cysts in the newborn. J Pediatr Surg 30(1): 42-44.

\section{Your next submission with Juniper Publishers} will reach you the below assets

- Quality Editorial service

- Swift Peer Review

- Reprints availability

- E-prints Service

- Manuscript Podcast for convenient understanding

- Global attainment for your research

- Manuscript accessibility in different formats

( Pdf, E-pub, Full Tsext, Audio)

- Unceasing customer service

Track the below URL for one-step submission

https://juniperpublishers.com/online-submission.php 